\title{
Construction and simulation of a multi-reflection time-of-flight mass spectrometer at the University of Notre Dame
}

\author{
B.E. Schultz, J.M. Kelly, C. Nicoloff, J. Long, S. Ryan, M. Brodeur \\ University of Notre Dame, Department of Physics, 225 Nieuwland Science Hall, Notre Dame, IN 46556
}

\begin{abstract}
One of the most significant problems in the production of rare isotopes is the simultaneous production of contaminants, often time isobaric. Thus, a high-resolution beam purification method is required which needs to be compatible with both the low yield and short half-life of the desired radionuclide. A multi-reflection time-of-flight mass spectrometer meets all these criteria, in addition to boasting a smaller footprint relative to traditional separator dipole magnets. Such a device is currently under construction at the University of Notre Dame and is intended to be coupled to the IG-ISOL source of the planned cyclotron facility. The motivation and conceptual design are presented, as well as the status of simulations to determine the feasibility of using a Bradbury-Nielsen gate for bunching ion beams during initial system testing.
\end{abstract}

Keywords:

Multi-reflection time-of-flight mass separator, Isobar separator, Isobaric contamination

\section{Introduction}

Experiments involving radioactive nuclei often require pure samples of a single isobar. Typical magnetic separators, with resolving powers of $R<5000$, can easily separate species by mass number but lack the resolving power to eliminate isobaric contaminants. The multi-reflection time-of-flight mass spectrometer (MR-TOF-MS) [1] has become a popular choice to purify radioactive ion beams (RIB), with devices installed or under construction at several RIB facilities worldwide $[2,3,4$, 5, 6]. An MR-TOF-MS traps ions between two electrostatic mirrors, folding the flight path into an extremely compact device. In addition, the isochronous nature [1] of the MR-TOFMS creates a sharp time focus, resulting in resolving powers on the order of $10^{5}$. In addition to a small physical footprint, the MR-TOF is relatively simple and inexpensive to construct. With typical ion flight times of $\leq 10 \mathrm{~ms}$, the method is compatible with short half-life and low-yield nuclei, which are of great interest at RIB facilities. The MR-TOF-MS can also fill the role of a mass spectrometer, permitting mass measurements on short-lived nuclei with precisions comparable to those obtained using Penning traps $[7,8,9,10]$.

An MR-TOF-MS is currently under construction at the University of Notre Dame Nuclear Science Laboratory. A series of simulations are also being performed to help guide the final design of the offline test setup and assess the performance of the MR-TOF-MS, in preparation for commissioning. The MRTOF-MS is intended to be installed in the planned Notre Dame Cyclotron Facility [11] to perform isobaric purification of RIB

Email address: bschult4@nd.edu (B.E. Schultz) for nuclear physics experiments [12]. The design and operation of the device is described below, as well as the status of assembly and simulations.

\section{MR-TOF-MS Design and Offline Test Setup}

The mirror electrode size and spacing of the MR-TOF-MS is based on the device installed [13] at ISOLDE/CERN, but with a different assembly design. The trap consists of two identical electrostatic ion mirrors separated by a drift tube, as shown in Figure 1 (top). Each mirror has five stainless steel electrodes and an einzel lens with a total length of $155 \mathrm{~mm}$. The mirror section is capped and shielded on both ends by grounded electrodes. Electrical isolation between adjacent electrodes is accomplished by $9.5 \mathrm{~mm}$ diameter ceramic balls, socketed into conical holes on the electrode faces. The entire trap structure is screwed together under compression, which along with tight machining tolerances minimizes the axial misalignment of the electrodes. Figure 2 shows an assembled mirror section.

The central drift tube has a length of $480 \mathrm{~mm}$, giving a total trap length of $790 \mathrm{~mm}$. The drift tube will be utilized to trap and extract ions using the so-called "in-trap lift method" [14], where the drift tube is pulsed from high voltage to ground as the ions pass through it, trapping them. The electrodes are mounted on segmented rods which run the length of the device.

A schematic of the MR-TOF-MS offline test setup is shown in Figure 1 (bottom). A stable surface ion source from HeatWave Labs [15] will be used for offline testing. Initially, a ${ }^{40} \mathrm{Ca}$ source will be used, which inherently comes with potassium contaminants. Separation of the ${ }^{40} \mathrm{Ca} /{ }^{40} \mathrm{~K}$ mass doublet requires a resolving power of $\approx 28,000$, making it an ideal case for initial testing. The continuous beam from the source will be 


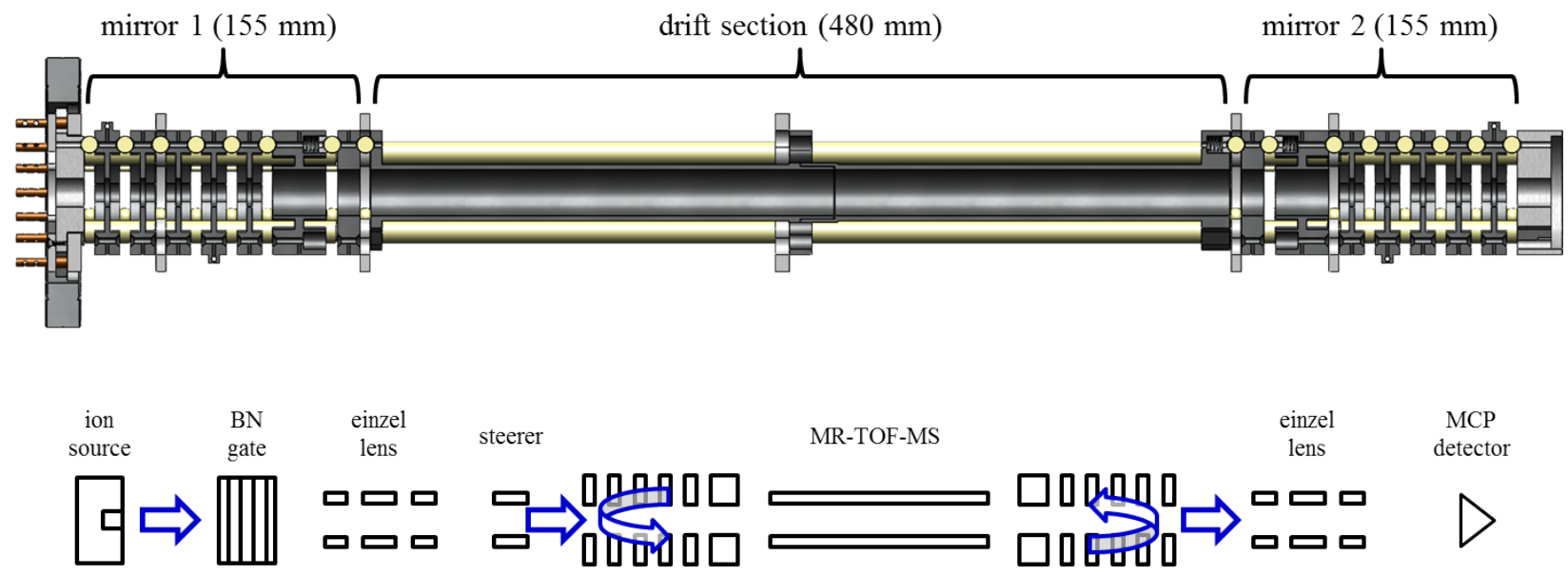

Figure 1: (Top) MR-TOF-MS trap cross section. (Bottom) Schematic of offline test setup and electrode configuration used for SIMION simulations (not to scale).

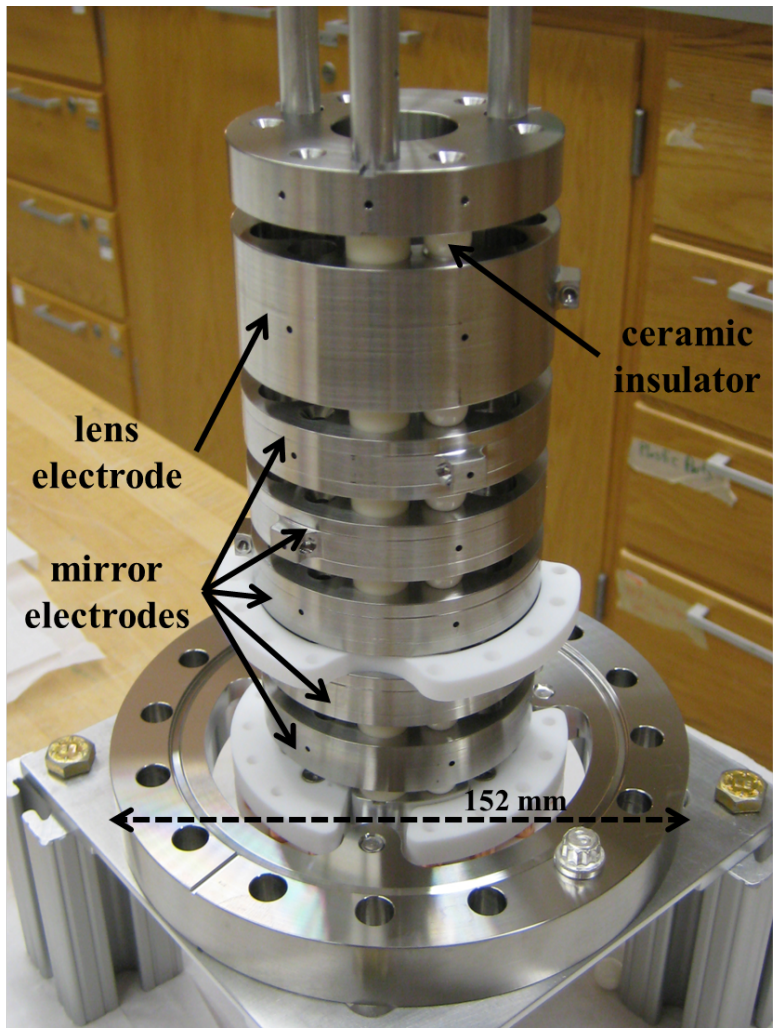

Figure 2: Assembled mirror section. chopped using a Bradbury-Nielsen gate (BNG) [16] connected to a fast switch, simulations of which will be discussed in the following section. The use of a surface source allows for the production of ion beams of well-defined energy with a minimal energy spread (0.24 eV FWHM) [17], while simulation shows only a small (but non-negligible) increase in energy spread from the use of the gate (see below). In the future, the BNG will be replaced by a radiofrequency quadrupole (RFQ) trap [18] for cooling and bunching of the ion beam produced by the IG-ISOL source. The BNG will then be moved to the extraction side of the trap to select the ion bunch of interest from the other separated species. An einzel lens and steerer are mounted downstream of the ion source to optimize injection into the trapping region, while a second einzel lens is mounted upstream of the trap to refocus the extracted ions.

The trap chamber and beamline are assembled from standard 6-inch CF components. Four $300 \mathrm{~L} / \mathrm{s}$ turbomolecular pumps (Agilent TwisTorr 304 FS) backed by an Agilent IDP-15 scroll pump will be used to evacuate the chamber and surrounding beamline. The pressure will be monitored using an InstruTech BA601 hot-cathode ion gauge and FlexRax 4000 controller. In the future, the presence of a buffer-gas-filled RFQ upstream of the MR-TOF-MS will impact the pressure inside the trap. Collisions with background gas will result in ion losses, requiring an operating pressure of $<10^{-7}$ mbar (where the mean free path should be longer than the total flight path) to maximize transfer efficiency. Therefore, a pressure of $\approx 10^{-9}$ mbar without gas flow is aimed for; the trap is designed to be UHV-compatible and will be baked to reach this level.

Figure 3 illustrates the proposed voltage control and data acquisition system for the MR-TOF-MS test setup. While the accuracy of the applied voltages is important, the voltage stability is critical. In order to achieve a resolving power of $R \approx 100,000$, voltage stabilization on the order of $10 \mathrm{ppm}$ is required [19]. Therefore, all electrodes will be biased using ISEG EHS-series high-precision HV modules $\left(\approx 1 \times 10^{-6}\right.$ stability $)$ mounted in an MPOD chassis. Each module has a common floating ground to minimize noise pickup. Additional temperature regulation may be required and will be investigated once 


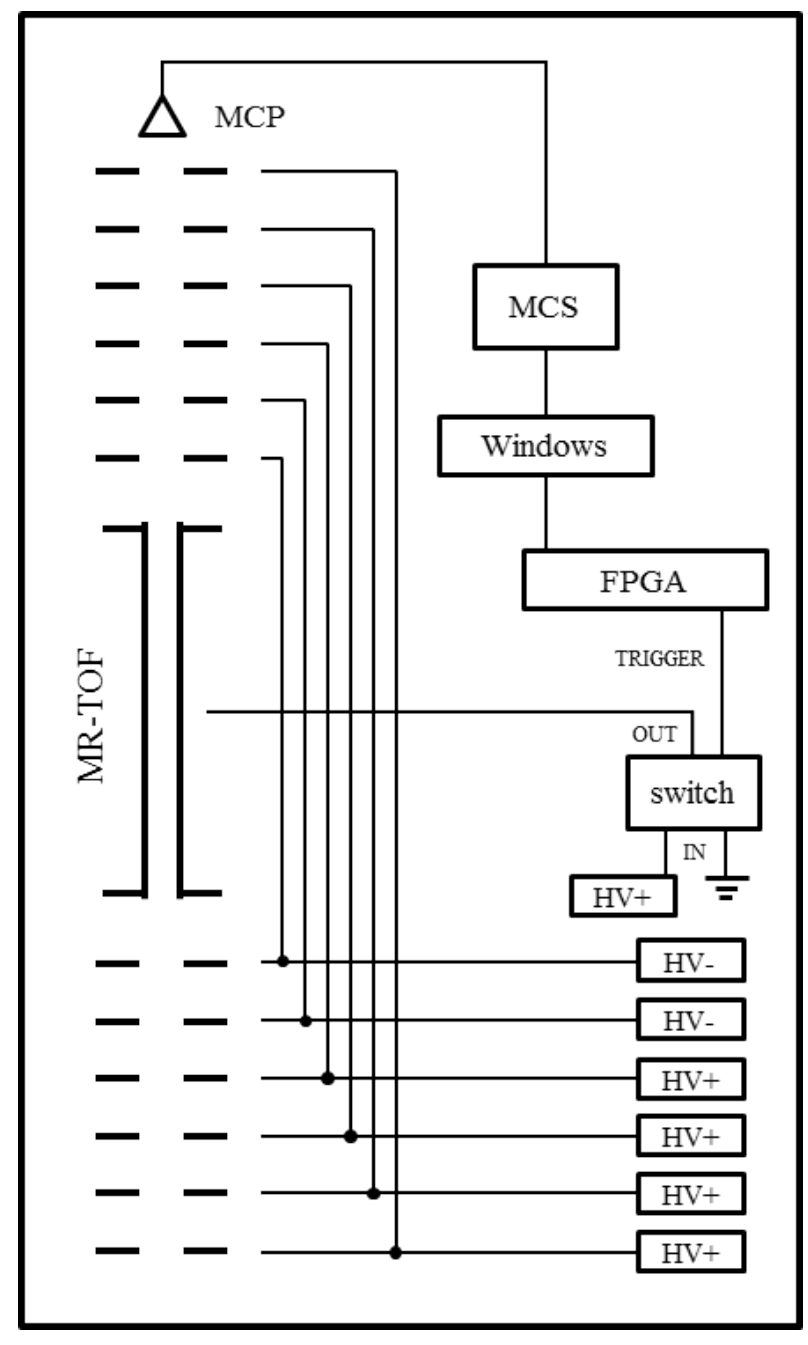

Figure 3: Schematic for MR-TOF-MS voltage control and data acquisition.

setup is complete. Output for the drift tube power supply will enter a high-voltage switch, facilitating trapping of ions as discussed above.

Power supply control and timing pulses for switching the $\mathrm{BN}$ gate and electrodes will be controlled by a National Instruments PXIe-7820R FPGA board using LabVIEW. Extracted ions will be detected on a $25 \mathrm{~mm}$ diameter microchannel plate (MCP) detector in the chevron configuration. Ion signals from the MCP detector will be recorded and analyzed using an oscilloscope card (National Instruments PXIe-5114, 250 mega-samples/s). To assist in tuning of the ion beam through the trap, the fluorescence from a phosphor screen mounted behind the MCP can be monitored using a CCD camera.

\section{Mirror Voltage and BNG Simulations}

Simulations related to the MR-TOF-MS that are being performed can be broken down into three types: mirror voltage optimization with ion bunches created at the center of the device, investigation of ion bunching using the BNG, and optimization of the MR-TOF-MS injection and trap voltages using

\begin{tabular}{cc}
\hline Electrode & Bias Voltage $(\mathrm{V})$ \\
\hline Mirror 1 & 3308.41 \\
Mirror 2 & 2306.80 \\
Mirror 3 & 1754.57 \\
Mirror 4 & 321.61 \\
Mirror 5 & -2131.92 \\
Lens & -4017.22 \\
\hline
\end{tabular}

Table 1: Optimized mirror voltages from simulations described in text. Mirror $1=$ electrode at end of trap, Mirror $2=$ second electrode from end, etc.

BNG-bunched beam. The following discussion will focus on the first two, as injection simulations are still ongoing. The simulations were performed using SIMION 8.1 [20].

\subsection{Optimizing Mirror Voltages}

The Notre Dame MR-TOF-MS mirror dimensions and electrode spacing were based on the ISOLTRAP device, but with a longer central lift electrode. Hence, ISOLTRAP's mirror voltages [3] were used as a starting point to optimize our device. This was done in a series of simulations using a gradient ascent algorithm. In this process, a spherical bunch of ions $(A / q=40)$ with a Gaussian density distribution $(\sigma=3 \mathrm{~mm})$ and an energy spread of zero was created at the center of the MR-TOF-MS. The ions had a fixed initial energy $(2000 \mathrm{eV})$ and direction and were allowed to cycle for up to 650 round trips, while their time-of-flight was recorded each time they crossed the midplane of the trap. The mass resolving power $R$ was calculated from the ratio of the average time-of-flight $(t)$ to the full-width half-maximum $(\Delta t)$, and is defined as $R=t /(2 \Delta t)$. The potential on a pair of electrodes was then varied, and the process was repeated. Once the potential on every pair of electrodes had been scanned, a new set of voltages was determined from the direction of the vector of greatest ascent. The resulting manydimensional gradient in "mirror-electrode space" indicated how the particular mirror potentials should be modified to converge to a maximum in $R$. (Note that this optimization process will find a local maximum, which will not necessarily equal the global value.) Figure 4 shows $R$ as a function of the number of round trips through the trap.

This process resulted in a maximum resolving power of $R_{F W H M} \approx 130,000$, corresponding to $\approx 650$ rounds trips and a trapping time of $\approx 10 \mathrm{~ms}$. The oscillatory behavior appears consistent with the compensation of aberrations after a 4-fold number of passes through the trap, as seen in isochronous mass separators $[19,21]$. For comparison, experimental resolving powers of $\approx 169,000$ for ${ }^{12} \mathrm{C}$ at RIKEN [19], $\approx 200,000$ for ${ }^{39} \mathrm{~K}$ at ISOLTRAP [3], and $\approx 600,000$ at GSI [22] have been achieved.

\subsection{Ion Bunching with a BNG}

In order to maximize the MR-TOF-MS resolving power, ions need to be injected with as little spread in energy and time as possible. At the planned cyclotron facility, an RFQ will fill this need; however, since this device has not yet been constructed, 


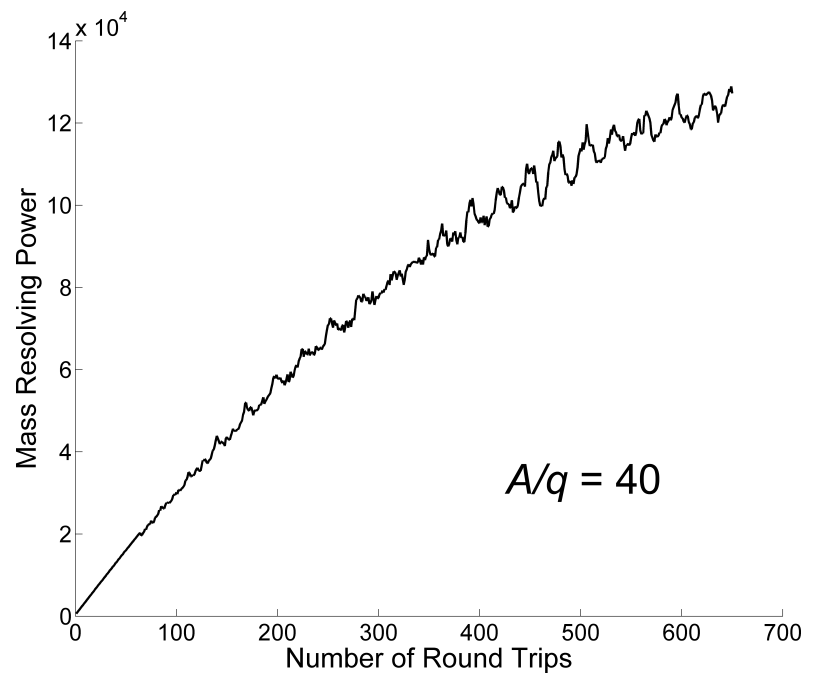

Figure 4: Dependence of resolving power on number of round trips through trap for ions with $A / q=40$. The trapping time for 650 revolutions is $\approx 10 \mathrm{~ms}$.

we have investigated the possibility of using a BNG to create ion bunches. To this end, the effect of switching the gate voltage on the time and energy spread was simulated.

The gate consists of two sets of 27 parallel wires, $50 \mu \mathrm{m}$ in diameter each and a separation of $0.56 \mathrm{~mm}$ between adjacent wires (1.12 $\mathrm{mm}$ between wires of the same voltage). In order to simulate a continuous ion beam, the code created ions over a time interval longer than (and centered around) the period in which the gate was open. Therefore, only the ions reaching the wire grid while the deflection voltages were set to zero would pass through and be recorded on the other side. Figure 5 illustrates a section of the geometry used in the simulation, and shows the ion trajectories when the BNG is open (a) and closed (b).

A custom-built high-voltage switch (rated to $\pm 800 \mathrm{~V}$ ) was attached to the BNG and the rise (fall) times to zero volts were measured to be $27 \mathrm{~ns}(7 \mathrm{~ns})$ and $15 \mathrm{~ns}(9 \mathrm{~ns})$ from $+300 \mathrm{~V}(-300$ $\mathrm{V})$, respectively. These values were used in the simulation to approximate the switching behavior of the grid. Bunches were given a width of $100 \mathrm{~ns}$, where the width is measured from the time when both wires reach $0 \mathrm{~V}$. Ions with $A=40$ were used to match initial testing conditions. The particles were given an initial Gaussian energy distribution centered at 4,600 eV with a FWHM of $0.24 \mathrm{eV}$, typical of surface ion sources like the one which will be used in system testing.

Figure 6(a) shows the voltage pulses applied to the BNG grid in the simulation $(V= \pm 1050 \mathrm{~V})$ to generate a $100 \mathrm{~ns}$ bunch width, and Figure 6(b) shows the time-of-flight of the resulting ion bunch. Large voltages were required to fully deflect the ions as the simulation "detector" had to be placed close to the gate due to geometric limitations, and should be considered a worstcase scenario. In reality, the applied voltages only need to be large enough to generate a deflection angle sufficient to prevent the deflected ions from being refocused by the Einzel lens downstream of the BNG. The effect on the ion energy is plotted in Figure 6(c). As seen in the graph, the switching increases the

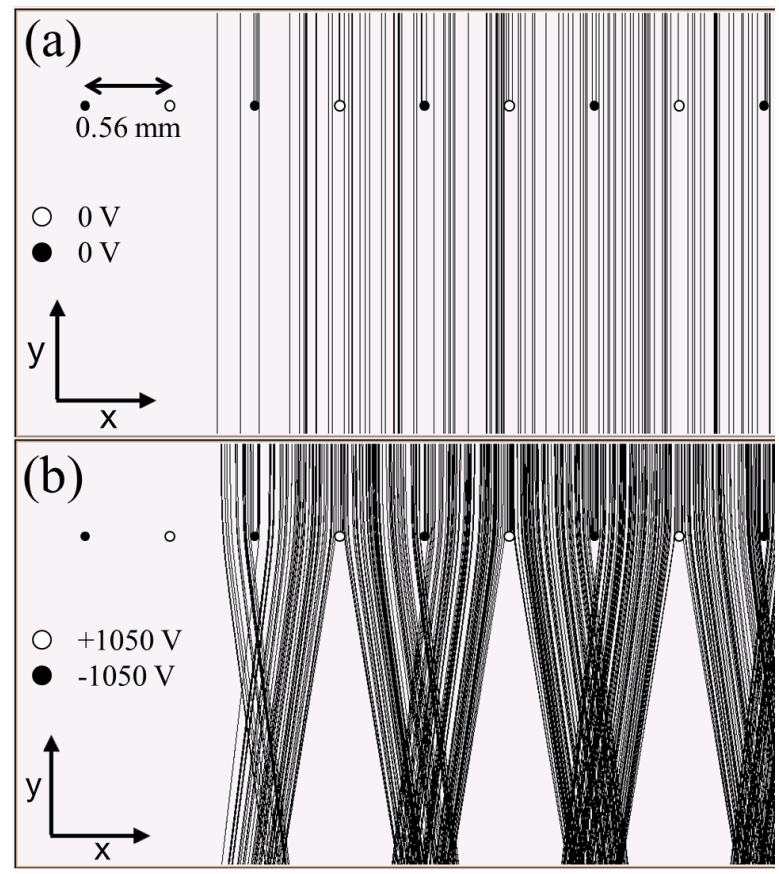

Figure 5: Ions $(A=40, E=4,600 \mathrm{eV})$ passing through section of BNG wire grid. (a) Gate open, with $0 \mathrm{~V}$ applied to both sets of wires. (b) Gate closed, with $+1050 \mathrm{~V}$ applied to one set of wires and $-1050 \mathrm{~V}$ to the other.

energy spread of the ions to $\approx 28 \mathrm{eV}$. The peaks at either end of the energy distribution likely come from ions that are very close to the grid when it switches. For comparison, the group from the University of Greifswald obtained a FWHM energy spread of $45 \mathrm{eV}$ for their simulated ${ }^{14} \mathrm{~N} / \mathrm{CO}$ beams bunched by a $120 \mathrm{~mm}$ long pulsed drift tube [13]. In spite of this large energy spread, they were able to obtain a resolving power of $R \approx 80,000$. Although the spread induced by the BNG is larger than expected, it should prove sufficient to generate ion bunches that can reach the necessary $R$ to resolve ${ }^{40} \mathrm{Ca}$ and ${ }^{40} \mathrm{~K}$. Additional simulations are underway to better characterize the BNGgenerated bunches, including a study of the effect on the radial emittance of the beam.

\section{Conclusions}

An MR-TOF-MS is currently under construction at the University of Notre Dame. Trap design, fabrication, and assembly have been completed. Design of Einzel lenses and beam diagnostics are being finalized, and fabrication will begin shortly. Once finished, offline commissioning of the ion source, detectors, and control systems will begin. Simulations have been performed to help characterize the operation of the MR-TOFMS and the BNG. Results obtained by optimizing the mirror potentials resulted in a resolving power of $\approx 130,000$. The feasibility of using a BNG to bunch ions for injection into the MR-TOF-MS has been studied as well. Simulations show that relatively short pulses can be generated via fast switching of the gate voltages, with a small but non-negligible effect on the energy spread. Although the increase from $0.24 \mathrm{eV}$ to $28 \mathrm{eV}$ 

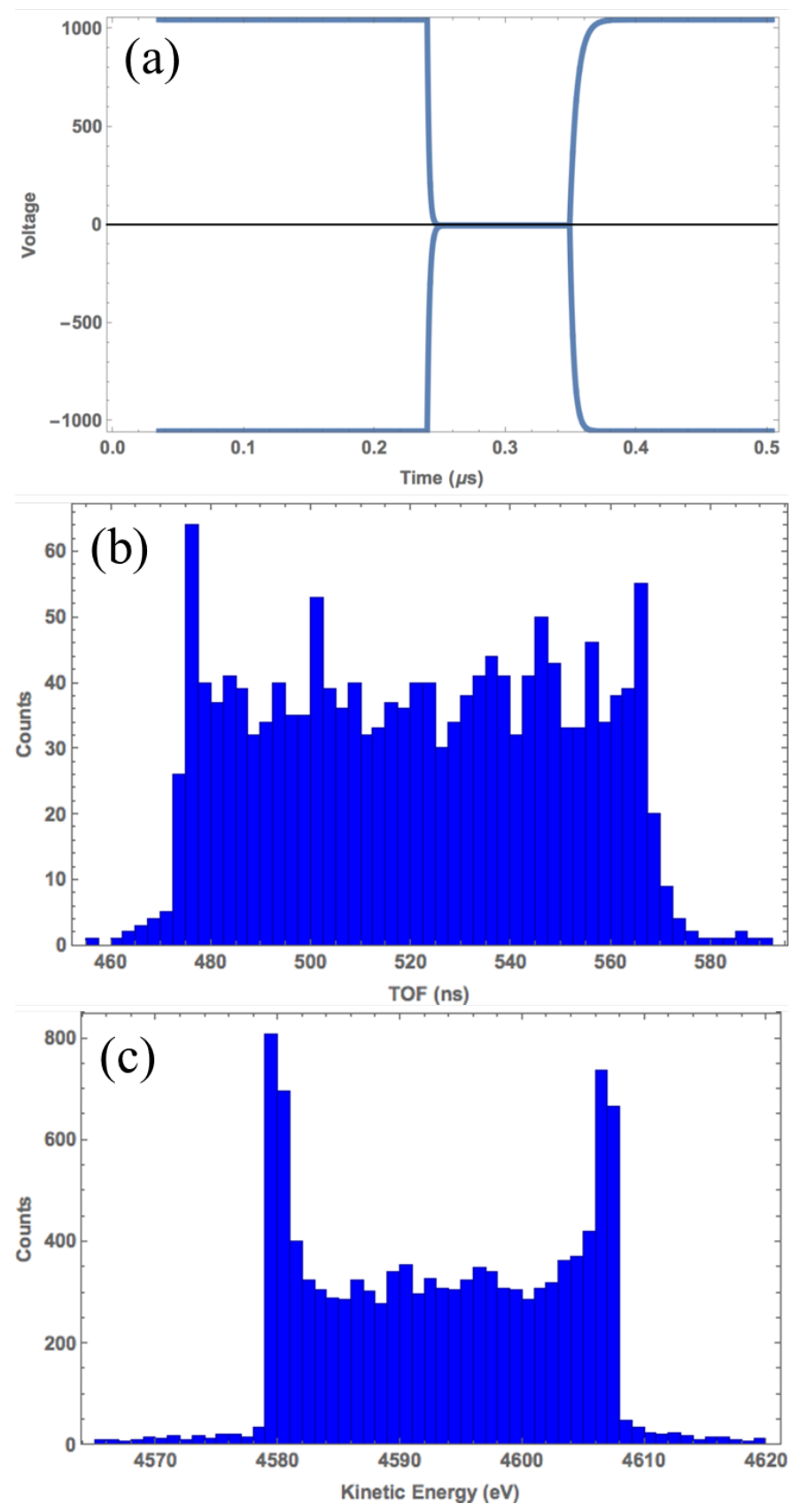

Figure 6: Bunch generation for ions with $A=40, q=+1$, and $E=4,600 \mathrm{eV}$. (a) BNG voltages used to generate a $100 \mathrm{~ns}$ wide bunch. (b) Number of counts versus time-of-flight for resulting bunch. (c) Switching generates an energy spread of $\Delta E \approx 28 \mathrm{eV}$. The range of each plot was truncated at the point when the counts per bin fell below five (each bin had a width of $1 \mathrm{eV}$ ). is larger than expected, it is smaller than the energy spread of the bunches used for commissioning of the MR-TOF-MS at Greifswald. As a result, the bunches generated using the BNG should be sufficient for initial commissioning of the Notre Dame MR-TOF-MS. However, during online operation, where the requirements on the resolving power are more strict, an RFQ cooler/buncher will be required to prepare the ions prior to injection into the MR-TOF-MS.

The authors would like to thank J. Brandon and D.J. Morrissey for providing the high-voltage switch to be used for the BNG. This work is supported by The National Science Foundation (NSF) grant number PHY-1419765.

[1] H. Wollnik and M. Przewloka, Int. J. Mass Spec. Ion Processes 96, 267 (1990).

[2] W.R. Plaß, T. Dickel, U. Czok, H. Geissel, M. Petrick, K. Reinheimer, C. Scheidenberger, M.I. Yavor, Nucl. Instrum. Methods B 266, 4560 (2008).

[3] R.N. Wolf, D. Beck, K. Blaum, Ch. Böhm, Ch. Borgmann, M. Breitenfeldt, F. Herfurth, A. Herlert, M. Kowalska, S. Kreim, D. Lunney, S. Naimi, D. Neidherr, M. Rosenbusch, L. Schweikhard, J. Stanja, F. Wienholtz, K. Zuber, Nucl. Instrum. Methods A 686, 82 (2012).

[4] A. Piechaczek, V. Shchepunov, H.K. Carter, J.C. Batchelder, E.F. Zganjar, S.N. Liddick, H. Wollnik, Y. Hu, B.O. Griffith, Nucl. Instrum. Methods B 266, 4560 (2008).

[5] W.R. Plaß, T. Dickel, C. Scheidenberger, Int. J. Mass Spectrom. 349, 134 (2013).

[6] T. Hirsh et al., Submitted to this proceeding.

[7] M. Rosenbusch, P. Ascher, D. Atanasov, C. Barbieri, D. Beck, K. Blaum, Ch. Borgmann, M. Breitenfeldt, R.B. Cakirli, A. Cipollone, S. George, F. Herfurth, M. Kowalska, S. Kreim, D. Lunney, V. Manea, P. Navrátil, D. Neidherr, L. Schweikhard, V. Somà, J. Stanja, F. Wienholtz, R.N. Wolf, K. Zuber, Phys. Rev. Lett. 114, 202501 (2015)

[8] W.R. Plaß, T. Dickel, S. Purushothaman, P. Dendooven, H. Geissel, J. Ebert, E. Haettner, C. Jesch, M. Ranjan, M.P. Reiter, H. Weick, F. Amjad, S. Ayet, M. Diwisch, A. Estrade, F. Farinon, F. Greiner, N. KalantarNayestanaki, R. Knöbel, J. Kurcewicz, J. Lang, I. Moore, I. Mukha, C. Nociforo, M. Petrick, M. Pfützner, S. Pietri, A. Prochazka, A.-K. Rink, S. Rinta-Antila, D. Scäfer, C. Scheidenberger, M. Takechi, Y.K. Tanaka, J.S. Winfield, M.I. Yavor, Nucl. Instrum. Methods B 317, 457 (2013).

[9] Y. Ito, P. Schury, M. Wada, S. Naimi, T. Sonoda, H. Mita, F. Arai, A. Takamine, K. Okada, A. Ozawa, H. Wollnik, Phys. Rev. C 88, 011306(R) (2013).

[10] F. Wienholtz, D. Beck, K. Blaum, Ch. Borgmann, M. Breitenfeldt, R.B. Cakirli, S. George, F. Herfurth, J.D. Holt, M. Kowalska, S. Kreim, D. Lunney, V. Manea, J. Menéndez, D. Neidherr, M. Rosenbusch, L. Schweikhard, A. Schwenk, J. Simonis, J. Stanja, R.N. Wolf, K. Zuber, Nature 498, 346 (2013).

[11] M. Brodeur, A. Aprahamian, D. Bardayan, J. Kelly, C. Nicoloff, D. Robertson, B.E. Schultz, JPS Conf. Proc. 6, 030113 (2015).

[12] M. Brodeur et al., Submitted to this proceeding.

[13] R.N. Wolf, M. Eritt, G. Marx, L. Schweikhard, Hyperfine Interact. 199, 115 (2011).

[14] R.N. Wolf, G. Marx, M. Rosenbusch, L. Schweikhard, Int. J. Mass Spec. 313, 8 (2012).

[15] HeatWave Labs, http://www.heatwavelabs.com/

[16] N.E. Bradbury, R.A. Nielsen, Phys. Rev. 49, 388 (1936).

[17] O. Heinz, R.T. Reaves, Rev. Sci. Instrum. 39, 1229 (1968).

[18] F. Herfurth, J. Dilling, A. Kellerbauer, G. Bollen, S. Henry, H.-J. Kluge, E. Lamour, D. Lunney, R.B. Moore, C. Scheidenberger, S. Schwarz, G. Sikler, J. Szerypo, Nucl. Instrum. Methods A 469, 254 (2001).

[19] P. Schury, M. Wada, Y. Ito, F. Arai, S. Naimi, T. Sonoda, H. Wollnik, V.A. Shchepunov, C. Smorra, C. Yuan, Nucl. Instrum. Methods B 335, 39 (2014).

[20] D.A. Dahl, Int. J. Mass Spec. 3, 200 (2000).

[21] H. Wollnik, Int. J. Mass Spec. Ion Proc. 131, 387 (1994).

[22] T. Dickel, W.R. Plaß, A. Becker, U. Czok, H. Geissel, E. Haettner, C. Jesch, W. Kinsel, M. Petrick, C. Scheidenberger, A. Simon, M.I. Yavor, Nucl. Instrum. Methods A 777, 172 (2015). 